\title{
Evaluation of dynamic thiol/disulfide redox state in community-acquired pneumonia
}

Ebru S. Parlak, MD, PhD, Murat Alisik, MD, PhD, Habibe Hezer, MD, PhD, Aysegul Karalezli, MD, PhD,

Hatice C. Hasanoglu, MD, PhD, Ozcan Erel, MD, PhD.

\begin{abstract}

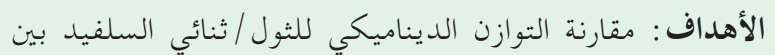

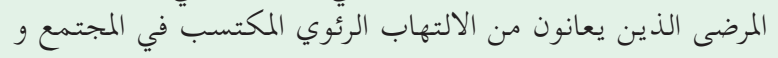
مجموعة الضبط.

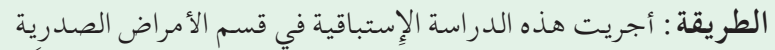

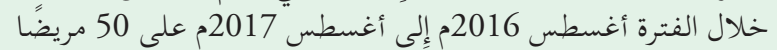

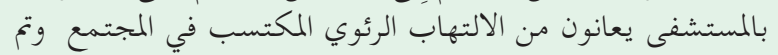

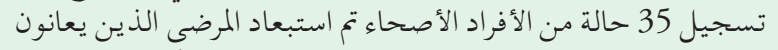

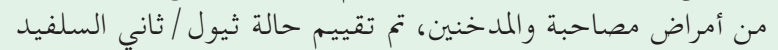

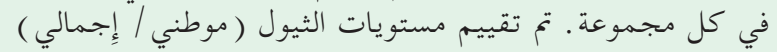

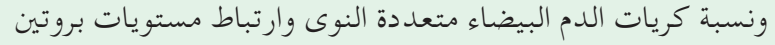

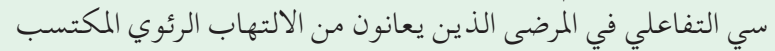
في المجتمع المعاعي في

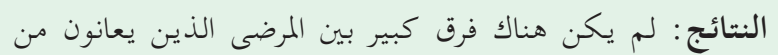

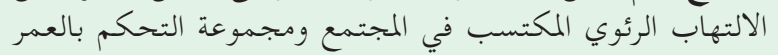
أو الجنس ( كلاهما،

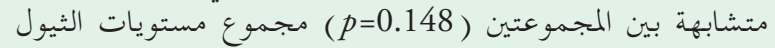
و الثيول الأصلي أقل بكثير كلاهما (

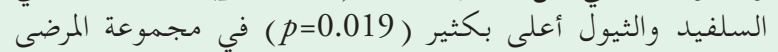

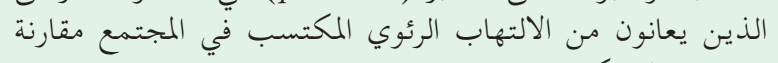

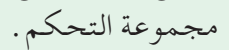

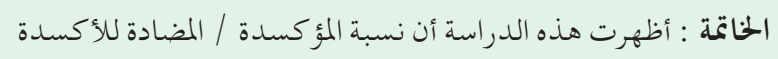

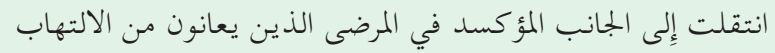

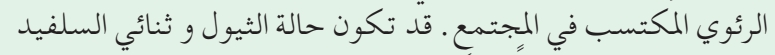

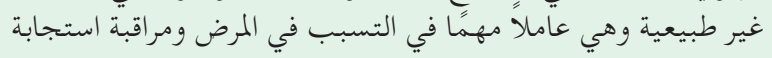

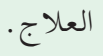

Objectives: To compare dynamic thiol/disulfide homeostasis between patients with community-acquired pneumonia (CAP) and healthy controls.

Methods: This prospective, case-control study was conducted in the Department of Pulmonary Diseases, Ankara Ataturk Training and Research Hospital, Yildirim
Beyazit University, Ankara, Turkey, between August 2016 and August 2017. In total, 50 hospitalized patients with $\mathrm{CAP}$ and 35 healthy individuals were enrolled. Patients with comorbidities and smokers were excluded. The thiol/disulfide state was evaluated in each group. Thiol levels (native/total) and \% polymorhonuclear leukocytes and C-reactive protein levels association were evaluated in patients with CAP.

Results: There was no significant difference between the CAP and control groups in age or gender (both, $p>0.05$ ). The disulfide (SS) levels were similar between the 2 groups $(p=0.148)$. The total thiol (TT) and native thiol $(\mathrm{SH})$ levels were significantly lower (all, $p=0.001$ ) and the SS/TT levels were significantly higher $(p=0.019)$ in the CAP group compared with the controls.

Conclusions: This study showed that the oxidant/ antioxidant ratio was shifted to the oxidative side in CAP patients. An abnormal thiol/disulfide state may be an important factor in the pathogenesis and monitoring the treatment response. The thiol resources may use for treatment in CAP and may positively affect the prognosis.

Saudi Med J 2018; Vol. 39 (5): $495-499$ doi: 10.15537/smj.2018.5.22111

From the Department of Pulmonary Diseases (Parlak, Hezer), Department of Clinical Biochemistry (Alisik), Ankara Ataturk Training and Research Hospital and from the Department of Clinical Biochemistry (Erel), Faculty of Medicine, Yildirim Beyazit University and from the Department of Pulmonary Diseases (Karalezli, Hasanoglu), Faculty of Medicine, Yildirim Beyazit University, Ankara, Turkey.

Received19th February 2018. Accepted 19th April 2018.

Address correspondence and reprint request to: Dr. Ebru S. Parlak, Department of Pulmonary Diseases, Ankara Ataturk Training and Research Hospital, Ankara, Turkey. E-mail: ebrusengulparlak@gmail.com 
P neumonia is a common cause of morbidity and mortality in adults worldwide. ${ }^{1}$ Among all infectious diseases, community-acquired pneumonia (CAP) is a leading cause of death in both the developed and developing worlds. ${ }^{2,3}$ Over the past 10 years, a $34 \%$ increase in hospital admissions has been observed, especially of elderly patients, due to CAP. ${ }^{4}$ Lungs are susceptible to oxidative damage due to their location and function. Oxidative stress and environmental oxidants resulting from an oxidant/antioxidant imbalance play an important role in respiratory tract diseases and inflammation. ${ }^{5}$ Plasma thiols have antiand pro-oxidant properties and contain a sulfhydryl group (-SH). ${ }^{6,7}$ The plasma thiol pool is comprised of albumin, protein thiols, and low molecular weight thiols (cysteine, cysteinylglycine, glutathione, homocysteine, and $\gamma$-glutamylcysteine). ${ }^{7}$ When thiol compounds undergo oxidation reactions, they form disulfide bonds. Dynamic thiol/disulfide homeostasis plays a critical role in metabolism and homeostasis. ${ }^{6,8,9}$ Recently, a variety of biomarkers have been examined in the serum of CAP patients. ${ }^{4}$ Many studies have focused on acute phase proteins. ${ }^{10}$ However, few studies have shown the importance of oxidative stress in the etiopathogenesis of pneumonia.

The aim of this prospective, case-control study was to compare dynamic thiol/disulfide homeostasis between patients with CAP and healthy controls.

Methods. This prospective, case-control study was conducted in the Department of Pulmonary Diseases, Ankara Ataturk Training and Research Hospital, Yildirim Beyazit University, Ankara, Turkey, between August 2016 and August 2017. The study was approved by the Ethics Committee (approval No. 26379996/188) and informed consent was obtained from all participants. This study was conducted in accordance with the tenets of the Declaration of Helsinki.

In total, 50 hospitalized patients with CAP and 35 healthy individuals were enrolled. Healthy controls were randomly selected from healthy individuals who came to outpatient polyclinic of chest diseases for routine control. Exclusion criteria were patients with comorbidities, including cardiovascular, cerebrovascular, liver and rheumatic diseases, as well as intensive care patients, acute-on-chronic kidney injury,

Disclosure. Authors have no conflict of interests, and the work was not supported or funded by any drug company. malignancies, diabetes mellitus, immunosuppression were excluded. Patients using antioxidants, lipidlowering drugs, cigarettes, or vitamin supplements were also ineligible for inclusion, as were those younger than 18 years and pregnant women. The thiol/disulfide state was compared in both study groups. The correlations between the percentage polymorphonuclear leukocytes (\%PMNLs) and C-reactive protein (CRP) and thiol (native/total) levels were evaluated in patients with CAP.

Laboratory tests. Blood samples were collected and serum was separated by centrifugation at $1,800 \mathrm{~g}$ for 10 minutes and stored at $-80^{\circ} \mathrm{C}$ until analysis. The serum native and total thiol (TT) levels were measured using the method described by Erel and Neselioglu using a Roche cobas-c501 automated analyzer (Roche, Mannheim, Germany). Briefly, the native thiol (SH) levels in each sample ere measured by reacting the thiol groups with 5,5'-dithiobis-(2-nitrobenzoic) acid (DTNB). To measure the TT levels of the samples, the disulfide bonds (SS) were first reduced with sodium borohydride to form free 2 thiol groups. The remaining sodium borohydride was eliminated with formaldehyde to prevent the reduction of DTNB, and the thiol groups, including both reduced and $\mathrm{SH}$ groups, were determined spectrophotometrically at $412 \mathrm{~nm}$ after the reaction with DTNB. The amount of dynamic disulfides equals half of the difference between the TTs and SHs. After determining the native and TTs and amount of disulfides, the SS/TT, SS/SH, and SH/TT percentage ratios were calculated. ${ }^{6}$

The CRP levels were measured turbidimetrically with the CardioPhase hsCRP kit (Siemens Healthcare Diagnostics Products, Marburg, Germany) using a BNII Nephelometer Analyzer (Siemens, Munich, Germany).

The PMNLs were counted using a Sysmex XE-2100 automated hematology system (Sysmex, Kobe, Hyōgo, Japan).

Statisticalanalysis. IBM SPSS Statistics for Windows, Version 20.0. (IBM, Armonk, NY, USA) was used for the statistical analyses. The normality of distribution of the data was evaluated with the Kolmogorov-Smirnov test. Variables were compared using the mean and standard deviation for continuous data. Student's t-test was used to evaluate differences between group means for continuous variables. The chi-square test $\left(x^{2}\right)$ was used to evaluate differences for categorical variables. A $p$-value $\leq 0.05$ was taken to indicate significance. To assess the associations between \%PMNLs and CRP and the thiol/disulfide parameters, correlation analysis was performed. 
Results. The study population comprised 50 patients with CAP (mean age $52.64 \pm 17.78$ years) and 35 healthy controls (mean age $47.37 \pm 9.83$ years). There was no significant difference between the CAP and control groups in terms of age or gender (both, $p>0.05)$. The TT and SH levels were significantly lower (all $p \leq 0.05$ ), and the SS/TT levels were significantly higher $(p \leq 0.05)$ in the CAP group compared with the controls. Table 1 summarizes the demographic data and laboratory findings of the CAP and control groups. Figure 1 shows the distribution of the TT levels $(\mu \mathrm{mol} / \mathrm{L})$ in the 2 groups. The correlations between the thiol/SS parameters and \%PMNLs and CRP were evaluated in the CAP group. Correlation analysis showed significant negative correlations between the TT levels $(p=0.005, \mathrm{r}=-0.389)$ and SH $(p=0.004, \mathrm{r}=$ $-0.402)$ and \%PMNLs and the TT levels $(p=0.002$, $\mathrm{r}=-0.421)(p=0.009, \mathrm{r}=-0.365)$ and SH $(p=0.865$, $\mathrm{r}=0.027)$ and CRP $(\mathrm{mg} / \mathrm{L})$.

Discussion. This is the first study to evaluate thiol/ disulfide homeostasis in patients with CAP. Thiols are antioxidant molecules that play an important role in the extracellular defense system. ${ }^{6,711}$ In the presence of oxidant molecules, proteins in cell walls and extracellular liquid become oxidized by the cysteine residue of the thiols. ${ }^{12}$ Thiols reveal disulfide bonds via this reaction. The oxidation of thiols is reversible. ${ }^{6}$ The thiol/disulfide balance entails that the physiological activities of the cell cycle play an important role. ${ }^{13}$ Many diseases are associated with oxidative stress, including malignancies, cardiovascular disease, and neurodegenerative disease. ${ }^{12}$

Table 1 - Demographic data and laboratory parameters in the CAP and control groups.

\begin{tabular}{|c|c|c|c|}
\hline Variable & CAP $(n=45)$ & Controls $(n=39)$ & $P$-value \\
\hline Gender $n(\%)$ & & & 1.000 \\
\hline Female & $18(36)$ & $13(37.1)$ & \\
\hline Male & $64(64)$ & $22(62.9)$ & \\
\hline Age (years) & $52.64 \pm 17.78$ & $47.37 \pm 9.83$ & 0.116 \\
\hline BMI $\left(\mathrm{kg} / \mathrm{m}^{2}\right)$ & $27.52 \pm 2.44$ & $26.74 \pm 3.10$ & 0.232 \\
\hline Total thiol $(\mu \mathrm{mol} / \mathrm{L})$ & $334.76 \pm 72.58$ & $514.99 \pm 40.68$ & 0.001 \\
\hline $\mathrm{SH}(\mu \mathrm{mol} / \mathrm{L})$ & $298.27 \pm 72.96$ & $472.50 \pm 44.78$ & 0.001 \\
\hline $\mathrm{SS}(\mu \mathrm{mol} / \mathrm{L})$ & $18.24 \pm 10.08$ & $21.24 \pm 8.08$ & 0.148 \\
\hline SS/TT (\%) & $5.60 \pm 3.30$ & $4.16 \pm 1.63$ & 0.019 \\
\hline \multicolumn{4}{|c|}{$\begin{array}{l}\text { CAP - community-acquired pneumonia, BMI - body mass index, } \\
\text { SH - native thiol, TT - total thiol, SS - disulfide, } p \leq 0.05 \text { significant } \\
\text { results }\end{array}$} \\
\hline
\end{tabular}

A standard approach for evaluating the response of biological systems to oxidative stress involves measuring the increase or decrease of a redox-sensitive molecule. ${ }^{14}$ There are few studies in the literature regarding the oxidant/antioxidant ratio in CAP. Duflo et $\mathrm{al}^{15}$ found that in plasma and bronchoalveolar lavage fluid, the levels of thiobarbituric acid reactant substances (TBARS), which constitute an index of the degree of oxidative damage, were increased significantly in patients who developed ventilator-associated pneumonia (VAP) compared with a non-VAP group. Moreover, alveolar glutathione peroxidase concentrations were lower in VAP. Trefler et $\mathrm{al}^{16}$ showed that TBARS levels were higher in CAP patients than in controls. Katsoulis et $\mathrm{al}^{17}$ evaluated the total antioxidant status (TOS) of 30 patients with CAP. They found that decreased serum TOS is associated with CAP. Muravlyova et $\mathrm{al}^{18}$ found that malondialdehyde and reactive carbonyl derivatives were significantly lower in CAP patients than in controls. Castillo et al ${ }^{14}$ showed that the antioxidant capacity of plasma, as well as glutathione and glutathione disulfide levels, were lower than in controls. In this study, we found that the total and native thiol pools were lower in patients with CAP compared with healthy controls. Moreover, the SS/TT ratio was significantly higher in the CAP group. An increased disulfide/TT ratio indicates that the thiol/disulfide ratio had shifted towards disulfide bond formation, and that oxidative stress was increased. Therefore, in CAP, the thiol/disulfide redox balance system shifted to the side of disulfide bond formation with increased oxidation.

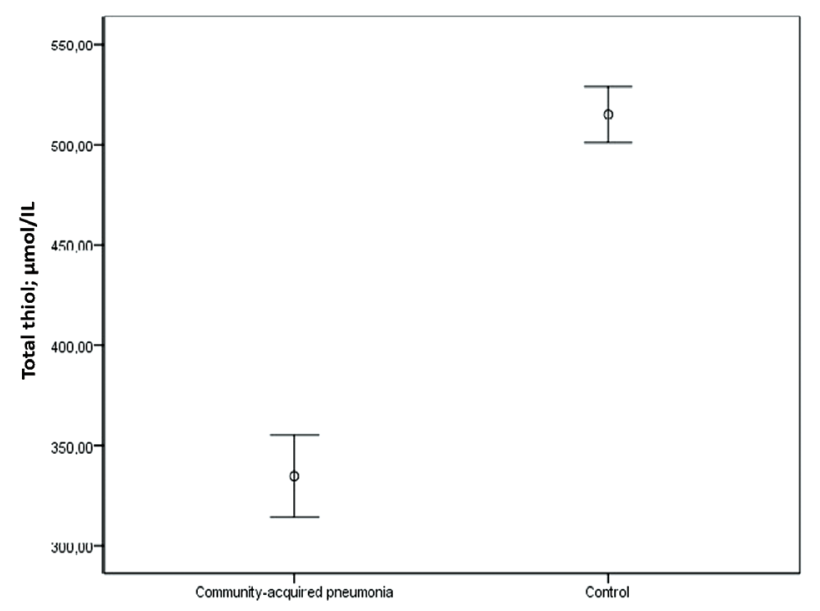

Figure 1 - Distribution of the total thiol levels ( $\mu \mathrm{mol} / \mathrm{L})$ in the community-acquired pneumonia (CAP) and control groups $(p=0.001)$. 
Lung cells are vulnerable to the detrimental effects of oxidants. ${ }^{19}$ In the normal lung, there is a balance between antioxidants and oxidants in the airway lining fluid and extracellular spaces, which helps to maintain normal physiological functions. ${ }^{20}$ In respiratory tract infections, an influx and activation of phagocytes into the lower airways, protecting against invading microorganisms, has been observed. ${ }^{14,21}$ Polymorphonuclear leukocytes and macrophages eliminate these microorganisms via reactive oxygen species (ROS) and lysosomal enzymes. An increased systemic ROS concentration may be seen in respiratory tract infections. ${ }^{14}$ Reactive oxygen species released from PMNLs and macrophages cause tissue damage in respiratory tract infections. ${ }^{21}$ Lung cells release inflammatory mediators and cytokines/chemokines in response to oxidative stress. ${ }^{19}$ The neutrophil percentage and CRP are important prognostic parameters in CAP. Curbelo et $\mathrm{al}^{22}$ found that higher CRP levels and neutrophil percentage predicted the 30-day mortality in CAP. We found negative correlations between the $\%$ PMNLs and thiol levels (native/total). We think that the ROS released from the increased PMNLs in CAP patients decrease the thiol levels by oxidizing the thiols. We also found a negative correlation between CRP and the thiol levels (native/total).

The results of the current study suggest that an abnormal thiol/disulfide ratio is important in the etiopathogenesis of CAP. Evaluation of thiol/disulfide parameters in CAP can make a significant contribution to assessing and monitoring patients with CAP. Moreover, thiol sources may be beneficial in stabilizing the oxidant/antioxidant ratio in CAP. N-acetyl cysteine (NAC) is an excellent source of sulfhydryl groups. ${ }^{23}$ Therefore, thiol sources such as NAC could contribute to CAP treatment.

Study limitations. The study population was small. Dynamic thiol disulfide homeostasis can be affected by various diseases and conditions. Therefore, many patients were excluded from the study because they had comorbid diseases.

More extensive studies are needed to determine the prognosis and importance of thiols in CAP.

Several biomarkers have been used to predict the prognosis of CAP. Most of these are acute-phase proteins. However, information on the importance of oxidative stress in the etiopathogenesis of pneumonia is lacking. This study showed that the oxidant/ antioxidant ratio shifted towards the oxidative side in CAP. Abnormal thiol/disulfide homeostasis could be an important factor in the pathogenesis of CAP and may be useful for evaluating the severity of the disease and treatment response. Future studies should investigate the importance of the thiol/disulfide redox state in the etiopathogenesis of CAP and answer the questions "Can thiol levels be used to predict the severity of CAP?" and "Are thiol resources useful for the treatment of CAP?"

Acknowledgment. We would like to thank Textcheck (www. textcheck.com) for the English language editing.

\section{References}

1. Aston SJ. Pneumonia in the developing world: Characteristic features and approach to management. Respirology 2017; 22: 1276-1287.

2. Aliberti S, Morlacchi LC, Faverio P, Fernandez-Botran R, Cosentini R, Mantero M, et al. Serum and exhaled breath condensate inflammatory cytokines in community-acquired pneumonia: a prospective cohort study. Pneumonia (Nathan) 2016; 23: 8 .

3. Shaddock EJ. How and when to use common biomarkers in community-acquired pneumonia. Pneumonia (Nathan) 2016; 28: 17.

4. Anevlavis S, Bouros D. Community acquired bacterial pneumonia. Expert Opin Pharmacother 2010; 11: 361-374.

5. Biswas SK, Rahman I. Environmental toxicity, redox signaling and lung inflammation: the role of glutathione. Mol Aspects Med 2009; 30: 60-76.

6. Erel O, Neselioglu S. A novel and automated assay for thiol/ disulphide homeostasis. Clin Biochem 2014; 47: 326-332.

7. Atmaca G. Antioxidant effects of sulfur-containing amino acids. Yonsei Med J 2004; 45: 776-788.

8. Biswas S, Chida AS, Rahman I. Redox modifications of proteinthiols: emerging roles in cell signaling. Biochem Pharmacol 2006; 71: 551-564.

9. Circu ML, Aw TY. Reactive oxygen species, cellular redox systems, and apoptosis. Free Radic Biol Med 2010; 48: 749-762.

10. Farah R, Makhoul N. Usefulness of various inflammatory markers to differentiate pulmonary edema from pneumonia. Isr Med Assoc J 2011; 13: 225-229.

11. Ozkan Y, Ozkan E, Simşek B. Plasma total homocysteine and cysteine levels as cardiovascular risk factors in coronary heart disease. Int J Cardiol 2002; 82: 269-277.

12. Go YM, Jones DP. Cysteine/cystine redox signaling in cardiovascular disease. Free Radic Biol Med 2011; 50: 495-509.

13. Turell L, Radi R, Alvarez B. The thiol pool in human plasma: the central contribution of albumin to redox processes. Free Radic Biol Med 2013; 65: 244-253.

14. Castillo RL, Carrasco RA, Alvarez PI, Ruiz M, Luchsinger $\mathrm{V}$, Zunino E, et al. Relationship between severity of adult community-acquired pneumonia and impairment of the antioxidant defense system. Biol Res 2013; 46: 207-213.

15. Duflo F, Debon R, Goudable J, Chassard D, Allaouchiche B. Alveolar and serum oxidative stress in ventilator-associated pneumonia. Br J Anaesth 2002; 89: 231-236. 
16. Trefler S, Rodríguez A, Martín-Loeches I, Sanchez V, Marín J, Llauradó $\mathrm{M}$, et al. Oxidative stress in immunocompetent patients with severe community-acquired pneumonia. A pilot study. Med Intensiva 2014; 38: 73-82.

17. Katsoulis K, Kontakiotis T, Baltopoulos G, Kotsovili A, Legakis IN. Total antioxidant status and severity of communityacquired pneumonia: are they correlated? Respiration 2005; 72 : 381-387.

18. Muravlyova L, Molotov-Luchankiy V, Bakirova R, Klyuyev D, Demidchik L, Lee V. Characteristic of the oxidative stress in blood of patients in dependence of community-acquired pneumonia severity. Open Access Maced J Med Sci 2016; 4: 122-127.

19. Rahman I, MacNee W. Oxidative stress and regulation of glutathione in lung inflammation. Eur Respir J 2000; 16: 534-554.
20. Crapo JD. Oxidative stress as an initiator of cytokine release and cell damage. Eur Respir J Suppl 2003; 44: 4-6.

21. Nowak D, Zieba M, Zawiasa D, Rozniecki J, Król M. Changes of serum concentration of lipid peroxidation products in patients with pneumonia. Monaldi Arch Chest Dis 1996; 51: 188-193.

22. Curbelo J, Luquero Bueno S, Galván-Román JM, OrtegaGómez M, Rajas O, Fernández-Jiménez G, et al. Inflammation biomarkers in blood as mortality predictors in communityacquired pneumonia admitted patients: Importance of comparison with neutrophil count percentage or neutrophillymphocyte ratio. Plos One 2017; 12: e0173947.

23. Mokhtari V, Afsharian P, Shahhoseini M, Kalantar SM, Moini A. A review on various uses of $\mathrm{N}$-acetyl cysteine. Cell J 2017; 19: 11-17. 